\title{
GENERALIZED GREEN'S EQUIVALENCES ON THE SUBSEMIGROUPS OF THE BICYCLIC MONOID
}

\author{
L. Descalço and P.M. Higgins \\ Department of Mathematical Sciences, University of Essex, \\ Wivenhoe Park, Colchester CO4 3SQ, United Kingdom
}

\begin{abstract}
We study generalized Green's equivalences on all subsemigroups of the bicyclic monoid $\mathbf{B}$ and determine the abundant (and adequate) subsemigroups of $\mathbf{B}$.
\end{abstract}

\section{INTRODUCTION}

The bicyclic monoid $\mathbf{B}$, is one of the most fundamental semigroups, with many remarkable properties and generalizations; see [1, 2, 6, 7, 8, 9, 10, 11, 12].

A description of the subsemigroups of the bicyclic monoid was obtained in [3], and by using this description several properties about all subsemigroups of $\mathbf{B}$ have been proved in [4] . In this paper we use this description to study the generalized Green's relations $\mathcal{L}^{*}$ and $\mathcal{R}^{*}$ of the subsemigroups of $\mathbf{B}$. This study is motivated by a J. Fountain's question, who asked if the description can be used to say which are the abundant and adequate subsemigroups of the bicyclic monoid.

Let $S$ be a semigroup and $a, b \in S$. We say that $a \mathcal{L}^{*} b$, if there is an oversemigroup of $S$ (a semigroup having $S$ as a subsemigroup) where $a \mathcal{L} b$. It is known and it is easy to check that (see [5]) $a \mathcal{L}^{*} b$ if and only if,

$$
\text { for all } x, y \in S^{1} \text { we have } a x=a y \Leftrightarrow b x=b y \text {. }
$$


The relation $\mathcal{R}^{*}$ is defined analogously as is the corresponding property. We say that a semigroup is abundant if every $\mathcal{L}^{*}$-class has an idempotent and every $\mathcal{R}^{*}$-class has an idempotent. An abundant semigroup is adequate if the set of its idempotents forms a semilattice.

The bicyclic monoid $\mathbf{B}$ is defined by the monoid presentation $\langle b, c \mid b c=1\rangle$; a natural set of unique normal forms for $\mathbf{B}$ is $\left\{c^{i} b^{j}: i, j \geq 0\right\}$ and we shall identify $\mathbf{B}$ with this set. The normal forms multiply according to the following rule:

$$
c^{i} b^{j} c^{k} b^{l}=\left\{\begin{array}{l}
c^{i-j+k} b^{l} \text { if } j \leq k \\
c^{i} b^{j-k+l} \text { if } j>k .
\end{array}\right.
$$

We are going to study the $\mathcal{L}^{*}$-classes and $\mathcal{R}^{*}$-classes of all subsemigroups of the bicyclic monoid in order to determine the abundant subsemigroups. We note that every set of idempotents from the bicyclic monoid is a semilattice (indeed a chain) and so a subsemigroup of the bicyclic monoid is adequate if and only if it is abundant.

We start by noting that two idempotents in the bicyclic monoid are always in separated $\mathcal{L}^{*}$-classes $\left(\mathcal{R}^{*}\right.$-classes). In fact, given two idempotents say, $c^{i} b^{i}, c^{j} b^{j}$ with $i<j$ we can use (1) choosing $x=c^{i} b^{i}$ and $y=c^{j} b^{j}$. We have $c^{j} b^{j} x=c^{j} b^{j} y=c^{j} b^{j}$ but $c^{i} b^{i} x=c^{i} b^{i}$ which is not equal to $c^{i} b^{i} y=c^{j} b^{j}$.

We will consider the different types of semigroups of $\mathbf{B}$ separately. Diagonal subsemigroups, one of the types, are formed by idempotents and so trivially are abundant. We begin by presenting some previous results giving the description of the subsemigroups of $\mathbf{B}$ in Section 2, then in Sections 3 and 4 we make some remarks that will be useful to study their $\mathcal{L}^{*}$-classes and $\mathcal{R}^{*}$-classes. Finally, in Sections 5 and 6 we consider the two relevant types of subsemigroups, the Upper and Two-sided subsemigroups, respectively.

\section{PREVIOUS RESULTS}

In this section we introduce the necessary notation and present the main result from [3] with the description of the subsemigroups of $\mathbf{B}$.

In order to define subsets of the bicyclic monoid it is convenient to see $\mathbf{B}$ as an infinite square grid, as shown in Figure 1. We start by introducing some 


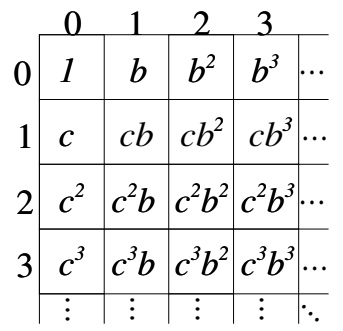

Figure 1: The bicyclic monoid

basic subsets of $\mathbf{B}$ :

$$
\begin{aligned}
& D=\left\{c^{i} b^{i}: i \geq 0\right\}-\text { the diagonal, } \\
& \left.L_{p}=\left\{c^{i} b^{j}: 0 \leq j<p, i \geq 0\right\}-\text { the left strip (determined by } p\right),
\end{aligned}
$$

for $p \geq 0$. For $0 \leq q \leq p \leq m$ we define the triangle

$$
T_{q, p}=\left\{c^{i} b^{j}: q \leq i \leq j<p\right\}
$$

Note that for $q=p$ this set is empty. For $i, m \geq 0$ and $d>0$ we define the rows

$$
\Lambda_{i}=\left\{c^{i} b^{j}: j \geq 0\right\}, \Lambda_{i, m, d}=\left\{c^{i} b^{j}: d \mid j-i, j \geq m\right\}
$$

and in general for $I \subseteq\{0, \ldots, m-1\}$,

$$
\Lambda_{I, m, d}=\bigcup_{i \in I} \Lambda_{i, m, d}=\left\{c^{i} b^{j}: i \in I, d \mid j-i, j \geq m\right\} .
$$

For $p \geq 0, d>0, r \in[d]=\{0, \ldots, d-1\}$ and $P \subseteq[d]$ we define the squares

$$
\begin{aligned}
& \Sigma_{p}=\left\{c^{i} b^{j}: i, j \geq p\right\}, \quad \Sigma_{p, d, r}=\left\{c^{p+r+u d} b^{p+r+v d}: u, v \geq 0\right\}, \\
& \Sigma_{p, d, P}=\bigcup_{r \in P} \Sigma_{p, d, r}=\left\{c^{p+r+u d} b^{p+r+v d}: r \in P ; u, v \geq 0\right\} .
\end{aligned}
$$

Pictures illustrating some of these sets can be found in [3].

The function $\rho: \mathbf{B} \rightarrow \mathbf{B}$ defined by $c^{i} b^{j} \mapsto\left(c^{i} b^{j}\right) \rho=c^{j} b^{i}$ is an antiisomorphism. Geometrically $\rho$ is the reflection with respect to the main diagonal.

We can now present the main result from [3]:

Proposition 2.1 Let $S$ be a subsemigroup of the bicyclic monoid. Then one of the following conditions holds: 
1. $S$ is a subset of the diagonal; $S \subseteq D$.

2. $S$ is a union of a subset of a triangle, a subset of the diagonal above the triangle, a square below the triangle and some rows belonging to a strip determined by the square and the triangle, or the reflection of such a union with respect to the diagonal. Formally there exist $q, p \in \mathbb{N}_{0}$ with $q \leq p, d \in \mathbb{N}, I \subseteq\{q, \ldots, p-1\}$ with $q \in I, P \subseteq\{0, \ldots, d-1\}$ with $0 \in P, F_{D} \subseteq D \cap L_{q}, F \subseteq T_{q, p}$ such that $S$ is of one of the following forms:

(i) $S=F_{D} \cup F \cup \Lambda_{I, p, d} \cup \Sigma_{p, d, P}$; or

(ii) $S=F_{D} \cup(F) \rho \cup\left(\Lambda_{I, p, d}\right) \rho \cup \Sigma_{p, d, P}$.

3. There exist $d \in \mathbb{N}, I \subseteq \mathbb{N}_{0}, F_{D} \subseteq D \cap L_{\min (I)}$ and sets $S_{i} \subseteq \Lambda_{i, i, d}(i \in I)$ such that $S$ is of one of the following forms:

(i) $S=F_{D} \cup \bigcup_{i \in I} S_{i}$; or

(ii) $S=F_{D} \cup \bigcup_{i \in I}\left(S_{i}\right) \rho$;

where each $S_{i}$ has the form

$$
S_{i}=F_{i} \cup \Lambda_{i, m_{i}, d}
$$

for some $m_{i} \in \mathbb{N}_{0}$ and some finite set $F_{i}$, and

$$
I=I_{0} \cup\left\{r+u d: r \in R, u \in \mathbb{N}_{0}, r+u d \geq N\right\}
$$

for some (possibly empty) $R \subseteq\{0, \ldots, d-1\}$, some $N \in \mathbb{N}_{0}$ and some finite set $I_{0} \subseteq\{0, \ldots, N-1\}$.

We call diagonal subsemigroups those defined by 1., two-sided subsemigroups those defined by 2., upper subsemigroups those defined by 3.(i) and lower subsemigroups those defined by 3.(ii). Pictures illustrating the several types of semigroups can be found in [3]. 


\section{$3 \quad \mathcal{L}^{*}$-CLASSES}

In general, to study the $\mathcal{L}^{*}$-classes of a subsemigroup $S$ of $\mathbf{B}$ we have to consider the equation $a x=a y$ appearing in (1), in our introductory section, and the following fact will be useful:

Lemma 3.1 We have ax = ay with $a=c^{i} b^{j}, x=c^{r} b^{s}, y=c^{u} b^{v} \in S, x \neq y$, i.e,

$$
c^{i} b^{j} c^{r} b^{s}=c^{i} b^{j} c^{u} b^{v}
$$

if and only if

$$
j \geq r, j \geq u \text { and } s-r=v-u \text {. }
$$

Proof. If $j \geq r, j \geq u$ and $s-r=v-u$ then $c^{i} b^{j} c^{r} b^{s}=c^{i} b^{j+s-r}=$ $c^{i} b^{j+v-u}=c^{i} b^{j} c^{u} b^{v}$. For the converse let's consider the four cases in the equation $c^{i} b^{j} c^{r} b^{s}=c^{i} b^{j} c^{u} b^{v}$. (i) $j \geq r, j \geq u$. In this case the equation becomes $c^{i} b^{j-r+s}=c^{i} b^{j-u+v}$ and so $s-r=v-u$ as stated. (ii) $j \geq r, j<u$. In this case we obtain $c^{i} b^{j-r+s}=c^{i-j+u} b^{v}$ and so we have $i=i-j+u$ (and $j-r+s=v$ ) which implies $j=u$, a contradiction. Analogously we cannot have (iii) $j<r, j \geq u$. (iv) Finally we show that is also not possible to have $j<r, j<u$. In this case the equation becomes $c^{i-j+r} b^{s}=c^{i-j+u} b^{v}$ which implies $r=u, s=v$ and so $x=y$, which contradicts the hypothesis.

Lemma 3.2 Let $c^{i} b^{j}, c^{k} b^{l} \in \mathbf{B}$, with $j \leq l$. If $c^{i} b^{j} x=c^{i} b^{j} y$ for some $x, y \in \mathbf{B}$ then $c^{k} b^{l} x=c^{k} b^{l} y$.

Proof. The statement holds trivially if $x=y$, so assume that $x \neq y$. Let $x=c^{r} b^{s}$ and $y=c^{u} b^{v}$. Since $c^{i} b^{j} c^{r} b^{s}=c^{i} b^{j} c^{u} b^{v}$ with $c^{r} b^{s} \neq c^{u} b^{v}$, using Lemma 3.1, we have $j \geq r, j \geq u$ and $s-r=v-u$. So, since $l \geq j \geq r$ and $l \geq j \geq u$, we have $c^{k} b^{l} c^{r} b^{s}=c^{k} b^{l-r+s}=c^{k} b^{l+v-u}=c^{k} b^{l} c^{u} b^{v}$.

As an immediate consequence of this fact, we just have to check one of the equivalences in (1):

Corollary 3.3 Two elements $c^{i} b^{j}, c^{k} b^{l}(j \leq l)$ in a subsemigroup $S$ of $\mathbf{B}$ are $\mathcal{L}^{*}$-related if and only if

$$
c^{k} b^{l} x=c^{k} b^{l} y \Longrightarrow c^{i} b^{j} x=c^{i} b^{j} y, \forall x, y \in S^{1} .
$$


Using this we can state a necessary and sufficient condition for two elements $A$ and $B$ in a subsemigroup of $\mathbf{B}$ to be $\mathcal{L}^{*}$-related, illustrated in Figure $2(x$ is in the horizontal shaded strip determined by the columns of $A$ and $B$ and $y$ in the shaded diagonal):

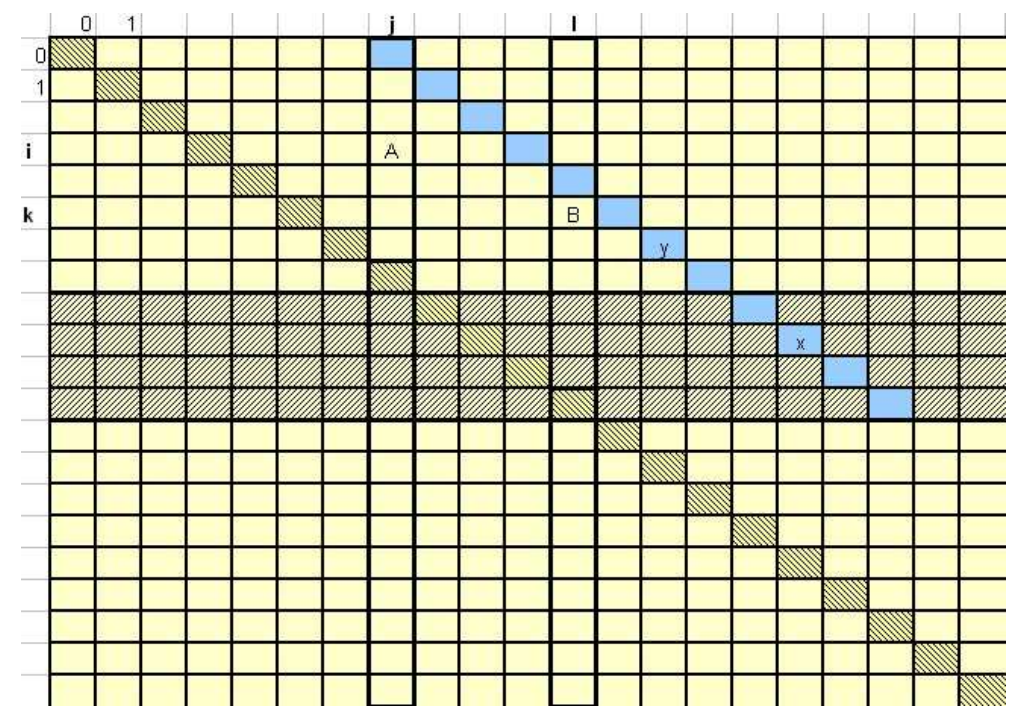

Figure 2: $\mathcal{L}^{*}$-relation in the subsemigroups of the bicyclic monoid

Lemma 3.4 Two elements $c^{i} b^{j}, c^{k} b^{l}(j \leq l)$ in a subsemigroup $S$ of $\mathbf{B}$ are not $\mathcal{L}^{*}$-related if and only if there exist two different elements $x=c^{r} b^{s}, y=c^{u} b^{v} \in$ $S$ such that $j<r \leq l, u \leq l$ and $s-r=v-u$.

Proof. Using Corollary 3.3, $c^{i} b^{j}$ and $c^{k} b^{l}$ are not $\mathcal{L}^{*}$-related if and only if there exist two elements $x, y \in S$ such that $c^{k} b^{l} x=c^{k} b^{l} y$ and $c^{i} b^{j} x \neq c^{i} b^{j} y$. Let $x=c^{r} b^{s}$ and $y=c^{u} b^{v}$. Using Lemma 3.1, $c^{k} b^{l} x=c^{k} b^{l} y$ is equivalent to $l \geq r, l \geq u$ and $s-r=v-u$, and $c^{i} b^{j} x \neq c^{i} b^{j} y$ is equivalent to $j<r \vee j<u \vee s-r \neq v-u$. Since $s-r=v-u$ it must be that $j<r \vee j<u$. We can assume, without loss of generality, that $j<r$, whence we have $j<r \leq l, u \leq l, s-r=v-u$.

As a trivial consequence we have the following useful sufficient condition for two elements to be $\mathcal{L}^{*}$-related: 
Lemma 3.5 Let $S$ be a subsemigroup of $\mathbf{B}$ and let $c^{i} b^{j}, c^{k} b^{l} \in S(j \leq l)$. If $S$ has no elements in rows $j+1, \ldots, l$ then $c^{i} b^{j} \mathcal{L}^{*} c^{k} b^{l}$.

And we have the following corollary:

Corollary 3.6 Two elements of a subsemigroup $S$ of $\mathbf{B}$ in the same column are $\mathcal{L}^{*}$-related.

This we knew already because two elements in the same column are $\mathcal{L}$ related in the bicyclic monoid.

Another consequence of Lemma 3.4 is the following:

Corollary 3.7 An $\mathcal{L}^{*}$-class of $S$ consists of a union of adjacent columns, i.e., there cannot exist two $\mathcal{L}^{*}$-related elements $A$ and $B$ and another element $C$ not $\mathcal{L}^{*}$-related to $A$ and $B$ in a column between them.

Proof. Let $A=c^{i} b^{j}, B=c^{k} b^{l}$ and $C=c^{m} b^{n}(j \leq n \leq l)$. If $A$ and $B$ are $\mathcal{L}^{*}$-related then, by Lemma 3.4, elements $x=c^{r} b^{s}, y=c^{u} b^{v}$ with $j<r \leq l, u \leq l$ and $s-r=v-u$ cannot exist. Hence such elements cannot exist with $j<r \leq n \leq l$ and $u \leq n$, so $C \mathcal{L}^{*} A \mathcal{L}^{*} B$ and $C \mathcal{L}^{*} B$ by transitivity.

\section{$4 \mathcal{R}^{*}$-CLASSES}

To obtain the corresponding facts for $\mathcal{R}^{*}$-classes we will use the standard antiisomorphism of an inverse semigroup $T$ to itself, $\rho: T \rightarrow T ; x \mapsto x^{-1}$. We note that $(x y) \rho=(x y)^{-1}=y^{-1} x^{-1}$. If $S$ is a subsemigroup of $T$, we denote by $S^{-1}$ the subsemigroup $S \rho$. If $T$ is the bicyclic monoid $\mathbf{B}$ then $\rho: \mathrm{B} \rightarrow \mathrm{B} ; c^{i} b^{j} \mapsto c^{j} b^{i}$ and

$$
\left(c^{i} b^{j} c^{k} b^{l}\right)^{-1}=c^{l} b^{k} c^{j} b^{i} .
$$

The following fact will be useful:

Lemma 4.1 If $S$ is a subsemigroup of an inverse semigroup $T$ and $a, b \in S$ then $(a, b) \in \mathcal{L}_{S}^{*}$ if and only if $\left(a^{-1}, b^{-1}\right) \in \mathcal{R}_{S^{-1}}^{*}$. 
Proof. Let $a, b \in S$. We have $(a, b) \in \mathcal{L}_{S}^{*}$ if and only if $a x=a y \Leftrightarrow b x=$ by $\left(\forall x, y \in S^{1}\right)$. This happens if and only if $(a x)^{-1}=(a y)^{-1} \Leftrightarrow(b x)^{-1}=$ $(b y)^{-1}\left(\forall x, y \in S^{1}\right)$. This is equivalent to $x^{-1} a^{-1}=y^{-1} a^{-1} \Leftrightarrow x^{-1} b^{-1}=$ $y^{-1} b^{-1}\left(\forall x, y \in S^{1}\right)$ what is the same as $u a^{-1}=v a^{-1} \Leftrightarrow u b^{-1}=v b^{-1}(\forall u, v \in$ $\left.\left(S^{-1}\right)^{1}\right)$ and so $\left(a^{-1}, b^{-1}\right) \in \mathcal{R}_{S^{-1}}^{*}$.

In the case where $T=S$ we have $S=S^{-1}$ and so we can say:

Lemma 4.2 If $S$ is an inverse semigroup and $a, b \in S$ then $(a, b) \in \mathcal{L}^{*}$ if and only if $\left(a^{-1}, b^{-1}\right) \in \mathcal{R}^{*}$.

Lemma 4.3 If $x a=y a$ with $a=c^{i} b^{j}, x=c^{r} b^{s}, y=c^{u} b^{v} \in S, x \neq y$, i.e,

$$
c^{r} b^{s} c^{i} b^{j}=c^{u} b^{v} c^{i} b^{j}
$$

then

$$
i \geq s, i \geq v \text { and } r-s=u-v .
$$

PROOF.

We have $x a=y a$ if and only if $(x a)^{-1}=(y a)^{-1}$. By (2), we have $(x a)^{-1}=$ $c^{j} b^{i} c^{s} b^{r}$ and $(y a)^{-1}=c^{j} b^{i} c^{v} b^{u}$ and so, by Lemma 3.1, we have $i \geq s, i \geq$ $v$ and $r-s=u-v$.

Lemma 4.4 Let $c^{i} b^{j}, c^{k} b^{l} \in \mathbf{B}, i \leq k$. If $x c^{i} b^{j}=y c^{i} b^{j}$ then $x c^{k} b^{l}=y c^{k} b^{l}$, for any $x, y \in \mathbf{B}, x \neq y$.

Proof. If $x c^{i} b^{j}=y c^{i} b^{j}$ then $\left(x c^{i} b^{j}\right)^{-1}=\left(y c^{i} b^{j}\right)^{-1}$. So $c^{j} b^{i} x^{-1}=c^{j} b^{i} y^{-1}$ and, by Lemma 3.2, $c^{l} b^{k} x^{-1}=c^{l} b^{k} y^{-1}$. Hence, $\left(x c^{k} b^{l}\right)^{-1}=\left(y c^{k} b^{l}\right)^{-1}$ and so $x c^{k} b^{l}=y c^{k} b^{l}$.

As an immediate consequence of this fact we have

Corollary 4.5 Two elements $c^{i} b^{j}, c^{k} b^{l}(i \leq k)$ in a subsemigroup $S$ of $\mathbf{B}$ are $\mathcal{R}^{*}$-related if and only if

$$
x c^{k} b^{l}=y c^{k} b^{l} \Longrightarrow x c^{i} b^{j}=y c^{i} b^{j}, \forall x, y \in S^{1} .
$$


The following lemma gives a necessary and sufficient condition for two elements to be $\mathcal{R}^{*}$-related and it is illustrated by Figure 3.

Lemma 4.6 Two elements $c^{i} b^{j}, c^{k} b^{l}(i \leq k)$ in a subsemigroup $S$ of $\mathbf{B}$ are not $\mathcal{R}^{*}$-related if and only if there exist two different elements $x=c^{r} b^{s}, y=c^{u} b^{v} \in$ $S$ such that $i<s \leq k, v \leq k$ and $r-s=u-v$.

Proof. The elements $c^{i} b^{j}, c^{k} b^{l}$ are not $\mathcal{R}_{S^{-}}^{*}$ related if and only if the elements $c^{j} b^{i}, c^{l} b^{k}$ are not $\mathcal{L}_{S^{-1}}^{*}$-related. By Lemma 3.4 this happens if and only if there exists $x^{-1}=c^{s} b^{r} \neq c^{v} b^{u}=y^{-1}$ in $S^{-1}$ such that $i<s \leq k, v \leq k$ and $r-s=u-v$. And so, if and only if, there exists $x=c^{r} b^{s} \neq c^{u} b^{v}=y$ in $S$ such that $i<s \leq k, v \leq k$ and $r-s=u-v$.

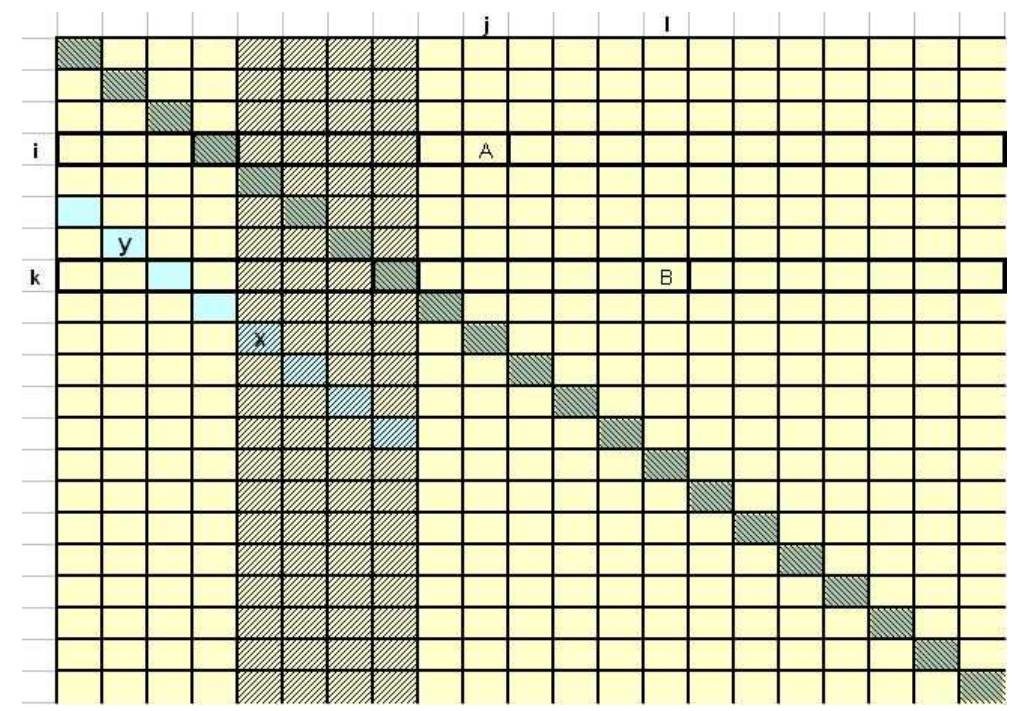

Figure 3: $\mathcal{R}^{*}$-relation in subsemigroups of the bicyclic monoid

Lemma 4.7 Let $S$ be a subsemigroup of $\mathbf{B}$ and let $c^{i} b^{j}, c^{k} b^{l} \in S(i \leq k)$. If $S$ has no elements in columns $i+1, \ldots, k$ then $c^{i} b^{j} \mathcal{R}^{*} c^{k} b^{l}$.

Corollary 4.8 Two elements of a subsemigroup $S$ of $\mathbf{B}$ in the same row are $\mathcal{R}^{*}$-related.

Corollary 4.9 An $\mathcal{R}^{*}$-class of $S$ consists of adjacent rows, i.e., there cannot exist two $\mathcal{R}^{*}$-related elements $A$ and $B$ in $S$ and another element $C$ in $S$ not related with $A$ and $B$ in a row between them. 
Proof. If $\left(c^{i} b^{j}, c^{k} b^{l}\right) \in \mathcal{R}_{S}^{*}$ then, by Lemma $4.1,\left(c^{j} b^{i}, c^{l} b^{k}\right) \in \mathcal{L}_{S^{-1}}^{*}$. By Corollary 3.7, $c^{j} b^{i}$ and $c^{l} b^{k}$ are in union of adjacent rows in $S^{-1}$, which means that $c^{i} b^{j}$ and $c^{k} b^{l}$ are in a union of adjacent columns in $S$.

\section{UPPER SUBSEMIGROUPS}

Upper semigroups may be abundant or not. A simple example is the free monogenic semigroup, generated by $b$, which is a non abundant upper semigroup, since it has no idempotents. We note that, since this semigroup is cancellative, it has a unique $\mathcal{L}^{*}$-class and a unique $\mathcal{R}^{*}$-class. If we adjoin the identity to it, we obtain the free monogenic monoid, which is an abundant upper subsemigroup of the bicyclic monoid, having one $\mathcal{L}^{*}$-class and one $\mathcal{R}^{*}$-class; both contain an idempotent, the identity of the monoid.

We start by considering finitely generated upper subsemigroups. They have the form $S=F_{D} \cup F \cup \Lambda_{I, m, d}$ where $I \subseteq \mathbb{N}_{0}, q=\min (I) \leq p=\max (I) \leq m$, $d \in \mathbb{N}, F_{D} \subseteq\left\{c^{i} b^{i}: i<q\right\}, F \subseteq\left\{c^{i} b^{j}: q \leq i \leq p, i \leq j<m\right\}$ are finite sets, and $\Lambda_{I, m, d}=\left\{c^{i} b^{j}: i \in I, d \mid j-i, j \geq m\right\}$ (see [4]). This semigroup is illustrated by Figure 4 . In this section we assume that $S$ is a semigroup of this kind.

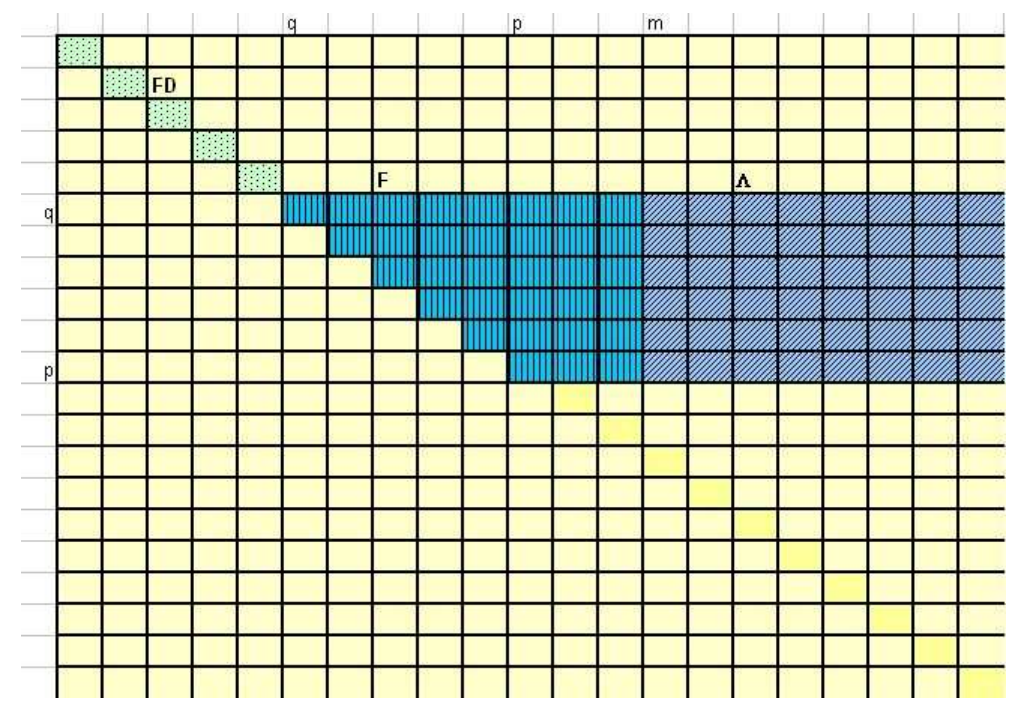

Figure 4: The region containing a semigroup $S=F_{D} \cup F \cup \Lambda_{I, m, d}$ 
We will first consider the case where $F_{D}=F=\emptyset$. In this case, $S=\Lambda_{I, m, d}$ is a finite union of special subsemigroups of $\mathbb{N}_{0}$ (numerical semigroups of the form $\left\{k d: k \in \mathbb{N}_{0}, k d \geq N\right\}$ with $d, N \in \mathbb{N}_{0}, d>0$ ). We will show that this subsemigroup has only one $\mathcal{L}^{*}$-class and only one $\mathcal{R}^{*}$-class. In fact, given two elements $c^{i} b^{j}, c^{k} b^{l} \in S$, with $j \leq l$, there are no elements of $S$ in rows $j+1, \ldots, l$ because $j+1>m$ and all elements of $S$ are in rows $q, \ldots, p$ with $p \leq m$. So using Lemma 3.5 we see that $c^{i} b^{j}, c^{k} b^{l}$ are $\mathcal{L}^{*}$-related. To see that there is also only one $\mathcal{R}^{*}$-class, we can take two arbitrary elements $c^{i} b^{j}, c^{k} b^{l} \in S$ with $i \leq k(\leq p \leq m)$. Since $S$ has no elements in columns $i+1, \ldots, k-1$, we cannot find two different elements $x, y$ in the conditions of Lemma 4.6, not even in the case where $p=m$. Hence $c^{i} b^{j}, c^{k} b^{l}$ are $\mathcal{R}^{*}$-related. Having only a $\mathcal{L}^{*}$-class and only a $\mathcal{R}^{*}$-class, for the subsemigroup to be abundant it just needs to contain one idempotent. This is only possible if $m=p$ and the idempotent $c^{p} b^{p}$ belongs to $S$. We summarize this in the following

Proposition 5.1 An upper subsemigroup $S$ of $\mathbf{B}$ of the form $S=\Lambda_{I, m, d}$ has a unique $\mathcal{R}^{*}$-class and a unique $\mathcal{L}^{*}$-class. It is abundant if and only if $m=p$ and $c^{p} b^{p} \in S$.

We consider now subsemigroups of the form $S=F_{D} \cup \Lambda_{I, m, d}$ where $F_{D}=$ $\left\{e_{1}, \ldots, e_{n}\right\}(n \geq 1)$. If $I$ has only one element, say $I=\{p\}$, then $S$ is in fact obtained starting from the numerical semigroup $\Lambda_{I, m, d}$ and adding successively the identities $e_{n}, e_{n-1}, \ldots e_{1}$. The elements of $\Lambda_{p, m, d}$ together with the idempotent of $S$ that is lower in the diagonal (which may be $c^{p} b^{p}$ ) form a cancellative monoid, hence having a unique $\mathcal{L}^{*}$-class and $\mathcal{R}^{*}$-class. Each other idempotent in $F_{D}$ is by itself an $\mathcal{L}^{*}$-class and an $\mathcal{R}^{*}$-class. So, if $c^{p} b^{p} \in S$ the classes are $\left\{e_{1}\right\},\left\{e_{2}\right\}, \ldots,\left\{e_{n}\right\}, \Lambda_{p, m, d}$. Otherwise the classes are $\left\{e_{1}\right\},\left\{e_{2}\right\}, \ldots,\left\{e_{n}\right\} \cup \Lambda_{p, m, d}$. In any case the subsemigroup is abundant.

Proposition 5.2 An upper semigroup of the form $S=F_{D} \cup \Lambda_{p, m, d}$ with $F_{D}=\left\{e_{1}, \ldots, e_{n}\right\} \quad(n \geq 1)$ is abundant. If $\Lambda_{p, m, d}$ has an idempotent the $\mathcal{L}^{*}$-classes and $\mathcal{R}^{*}$-classes are $\left\{e_{1}\right\},\left\{e_{2}\right\}, \ldots,\left\{e_{n}\right\}, \Lambda_{p, m, d}$. Otherwise they are $\left\{e_{1}\right\},\left\{e_{2}\right\}, \ldots,\left\{e_{n}\right\} \cup \Lambda_{p, m, d}$.

We continue with $S=F_{D} \cup \Lambda_{I, m, d}$ where $F_{D}=\left\{e_{1}, \ldots, e_{n}\right\}(n \geq 1)$ but assuming now that $I$ has more than one element. Let's first consider the case 
where $c^{p} b^{p} \in \Lambda_{I, m, d}(m=p)$. In this case, two elements $c^{i} b^{j}, c^{p} b^{k} \in \Lambda_{I, m, d}$ with $i<p$ are not $\mathcal{R}^{*}$-related since, letting $x$ be the lower idempotent in $F_{D}$ and $y=c^{p} b^{p}$, the elements $x$ and $y$ are in the conditions of Lemma 4.6. But any two elements $c^{i} b^{j}, c^{k} b^{l} \in \Lambda_{I, m, d}$ with $i, j<p$ are $\mathcal{R}^{*}$-related since $S$ has no elements in columns $q, \ldots, p-1$ and we can use Lemma 4.7. The lower idempotent in $F_{D}$, say $e=c^{i} b^{i}$, is $\mathcal{R}^{*}$-related to any element $c^{k} b^{l}$ in rows $I \backslash\{p\}$ because $k<p$ and $S$ has no elements in columns $i+1, \ldots, p-1$. The other idempotents in $F_{D}$ are $\mathcal{R}^{*}$-classes by themselves. So every $\mathcal{R}^{*}$-class has an idempotent. The $\mathcal{R}^{*}$-classes of these subsemigroups are illustrated by the example in Figure 5 .

Two elements $c^{i} b^{j}, c^{k} b^{l} \in \Lambda_{I, m, d}$ are $\mathcal{L}^{*}$-related because $j, l \geq p$, there are no elements in $S$ below row $p$ and so we can use Lemma 3.5. These $\mathcal{L}^{*}$-class contains already one idempotent, $c^{p} b^{p}$, and so the other idempotents in $F_{D}$ are $\mathcal{L}^{*}$-classes by themselves. Hence, also every $\mathcal{L}^{*}$-class has an idempotent and the semigroup is abundant.

The case where $c^{p} b^{p} \notin S$ can also be illustrated by Figure 5 , removing the last row. We show that, in this case, the set $\Lambda_{I, m, d}$ is an $\mathcal{L}^{*}$-class of $S$ without an idempotent, and so the semigroup is not abundant. The elements in $\Lambda_{I, m, d}$ are still $\mathcal{L}^{*}$-related. But the lower idempotent $c^{i} b^{i} \in F_{D}$ is not related to them, since, given $c^{k} b^{l} \in \Lambda_{I, m, d}$, we can find two elements $x=c^{j} b^{j+u d}, y=c^{k} b^{k+v d} \in$ $\Lambda_{I, m, d}$ in the same diagonal and in different rows, in the conditions of Lemma 3.4 .

Proposition 5.3 An upper semigroup of the form $S=F_{D} \cup \Lambda_{I, m, d}$ with $F_{D}=$ $\left\{e_{1}, \ldots, e_{n}\right\} \quad(n \geq 1)$ and $|I|>1$ is abundant if and only if $m=p$ and $c^{p} b^{p} \in$ $\Lambda_{I, m, d}$. In this case the $\mathcal{L}^{*}$-classes and $\mathcal{R}^{*}$-classes are $\left\{e_{1}\right\},\left\{e_{2}\right\}, \ldots,\left\{e_{n}\right\}, \Lambda_{I, m, d}$.

Finally we consider arbitrary finitely generated upper semigroups $S=F_{D} \cup$ $F \cup \Lambda_{I, m, d}$ where $F \neq \emptyset$. We note that, if $I$ has only one element, then $S$ is again obtained from a numerical semigroup adding finitely many idempotents and so we have:

Proposition 5.4 A subsemigroup of the form $F_{D} \cup F \cup \Lambda_{p, m, d}, F_{D}=\left\{e_{1}, \ldots, e_{n}\right\}$ $(n \geq 0)$ is abundant if and only if it contains at least one idempotent. If $c^{p} b^{p} \in S$ the $\mathcal{L}^{*}$-classes and $\mathcal{R}^{*}$-classes are $\left\{e_{1}\right\},\left\{e_{2}\right\}, \ldots,\left\{e_{n}\right\}, \Lambda_{I, m, d}$. Otherwise the classes are $\left\{e_{1}\right\},\left\{e_{2}\right\}, \ldots,\left\{e_{n}\right\} \cup \Lambda_{I, m, d}$. 


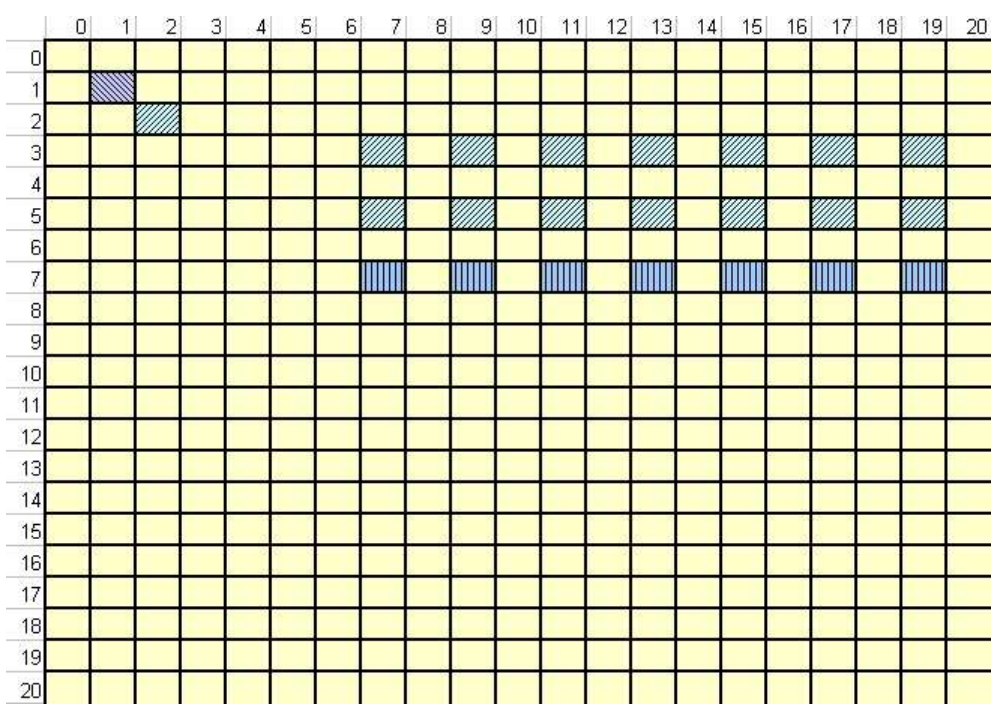

Figure 5: $\mathcal{R}$-classes of $S=F_{D} \cup \Lambda_{I, m, d}$

So we assume now that $I$ has a at least two elements. To identify the $\mathcal{L}^{*}$ classes of $S$, it is convenient to write $S=F_{D} \cup F^{\prime} \cup S^{\prime}$, where $F^{\prime}=S \cap\left\{c^{i} b^{j}\right.$ : $q \leq i \leq j<p\}$ and $S^{\prime}=S \cap\left\{c^{i} b^{j}: j \geq p\right\}$, as illustrated in Figure 6 .

The elements in $S^{\prime}$ are $\mathcal{L}^{*}$-related because they are on columns $p, p+1, \ldots$ and, since there are no elements in $S$ below row $p$, we can use Lemma 3.5. An element $c^{i} b^{j} \in F^{\prime}$ is not $\mathcal{L}^{*}$-related to an element $c^{k} b^{l} \in S^{\prime}$. In fact, since $S$ has elements in row $p$ and $i, j<p$, we can choose two different elements $x=c^{p} b^{p+u d}, y=c^{i} b^{i+v d}$ in the same diagonal, in the conditions of Lemma 3.4. Hence, if $F^{\prime} \neq \emptyset$ then $S^{\prime}$ is an $\mathcal{L}^{*}$-classe of $S$. Also in the case where $F^{\prime}=\emptyset$ the set $S^{\prime}$ is an $\mathcal{L}^{*}$-class of $S$. This is shown if $F_{D}=\emptyset$. And if $F_{D} \neq \emptyset$, we can see that the lower idempotent in $F_{D}$ is not $\mathcal{L}^{*}$-related say, with an element $c^{p} b^{k} \in S^{\prime}$ because we can choose two different elements $x=c^{p} b^{p+u d}$ and $y=c^{i} b^{i+v d}$ in $S$ with $i<p$ in the same diagonal, and use Lemma 3.4. So, in any case, $S^{\prime}$ is an $\mathcal{L}^{*}$-class of $S$ and for $S$ to be abundant it must contain the idempotent $c^{p} b^{p}$.

We continue the study of $\mathcal{L}^{*}$-classes considering now the elements in $F^{\prime}$, which are in finitely many columns. For the semigroup to be abundant, each $\mathcal{L}^{*}$-classe, that may be formed by the elements in one or more columns, must contain an idempotent. In Figure 7, we find an example of an abundant 


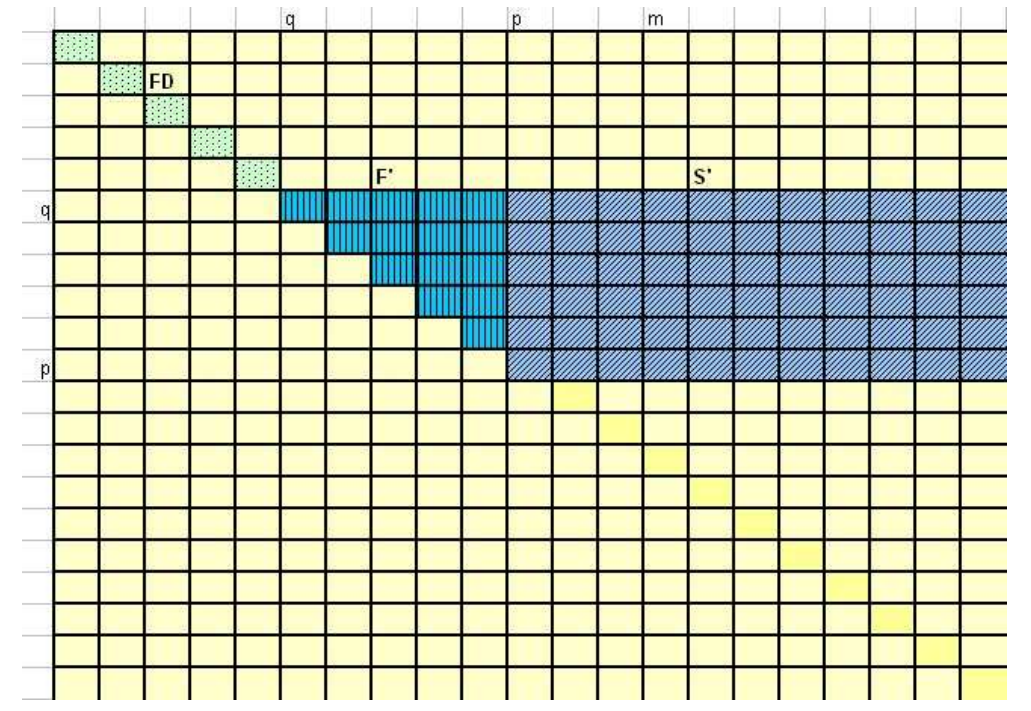

Figure 6: Determining the $\mathcal{L}^{*}$-classes of $S=F_{D} \cup F \cup \Lambda_{I, m, d}$

subsemigroup where some columns with elements do not have idempotents.

To check if all $\mathcal{L}^{*}$-classes of elements in $F_{D} \cup F^{\prime}$ have idempotents we just have to form unions with the $p-q$ columns. To do that, we start by observing that if two columns $i, j$ with elements with $q \leq i<j<p$, are in the same $\mathcal{L}^{*}$-class, then $c^{j} b^{j}$ cannot be in the class. In fact, if $c^{j} b^{j}$ were in the class then, given two elements $c^{k} b^{i}, c^{l} b^{j}$ we could obtain $x=c^{j} b^{j+u d}, y=c^{k} b^{k+v d}$ in the conditions of Lemma 3.4 and $c^{k} b^{i}, c^{l} b^{j}$ would not be related. So, the idempotent in an $\mathcal{L}^{*}$-class with elements from $F^{\prime}$ is either in the leftmost column or in $F_{D}$. Hence, to check if all classes have idempotents we can proceed the following way. We begin by forming a union of rows with elements, $L$, starting from first column $i \leq p-1$ with elements and going left. If we have already an idempotent we start forming next class. If not we add next column $j<i$ with elements to $L$, if there are no elements in rows $j+1, \ldots, i$. We proceed adding columns until no more columns can be added. After that, if the last column does not have an idempotent in $S$ (and there are still other columns with elements in $F^{\prime}$ on the left) we have found an $\mathcal{L}^{*}$-class without an idempotent. Otherwise $L$ is an $\mathcal{L}^{*}$-class with idempotent and we start forming the next class. After going through all columns of $F^{\prime}$ two things may happen. If the final union of rows $L$ has an idempotent then all $\mathcal{L}^{*}$-classes have idempotents. 


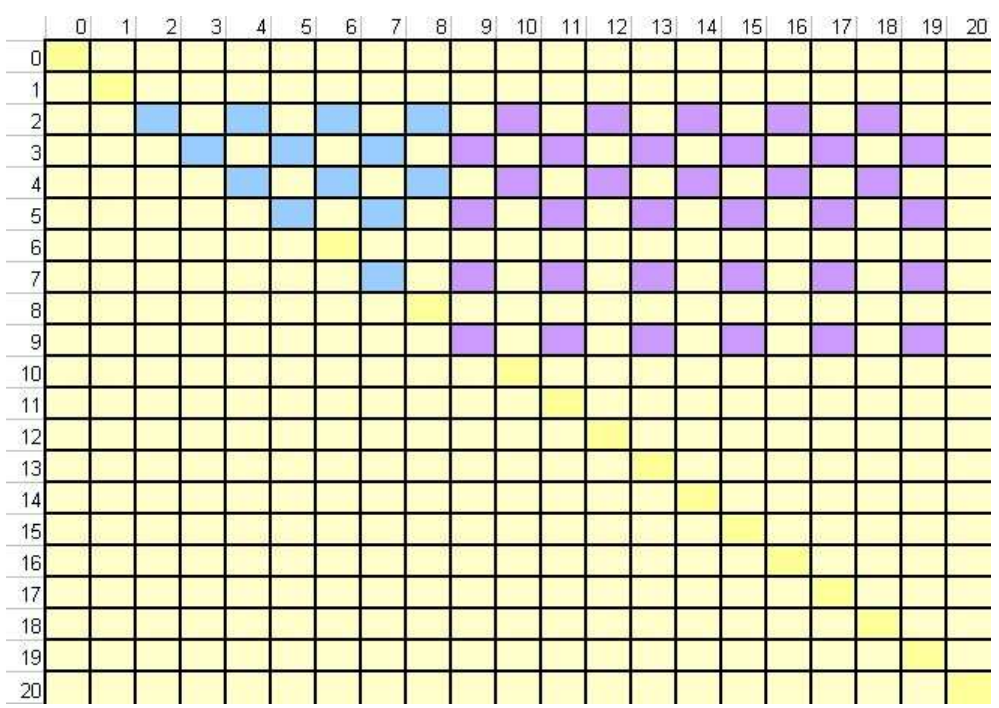

Figure 7: An abundant upper subsemigroup

If not, it may be possible that all $\mathcal{L}^{*}$-classes have idempotents if $F_{D} \neq \emptyset$. This can only occur if there is only one row in $S$ with elements in rows $k+1, \ldots, l$, where $k$ is the minimum of column indices in $L$ and $l$ is the maximum. In fact, we see using Lemma 3.4 that only in this case the lower idempotent in $F_{D}$ is $\mathcal{L}^{*}$-related to the elements in $L$.

To check if the $\mathcal{R}^{*}$-classes have idempotents we can proceed in a similar way. There are finitely many rows with elements and $\mathcal{R}^{*}$-classes are unions of adjacent rows. We start from row $i=p$ and go up adding rows and forming $\mathcal{R}^{*}$-classes. We consider the next row $j(=\max (I \backslash\{i\})$ in $I$. Using Lemma 4.6 we see that, elements in rows $i$ and $j$ are $\mathcal{R}^{*}$-related if and only if $S$ has no elements in columns $i+1, \ldots, j$ or, for each element $A$ in columns $i+1, \ldots, j$ we cannot find another element $\mathbf{B}$ in columns $0, \ldots, j$ in the same diagonal as $A$. Proceeding this way we can form the $\mathcal{R}^{*}$-classes, which are at most $|I|+\left|F_{D}\right|$, and check if they all have idempotents.

These algorithms allows us to check if a general upper subsemigroup of the form $F_{D} \cup F \cup \Lambda_{I, m, d}$ is abundant. Hence we can say the following

Proposition 5.5 Let $S=F_{D} \cup F \cup \Lambda_{I, m, d}$ be an upper subsemigroup of $\mathbf{B}$. Writing $S=F_{D} \cup F^{\prime} \cup S^{\prime}$, where $F^{\prime}=S \cap\left\{c^{i} b^{j}: q \leq i \leq j<p\right\}$ and $S^{\prime}=S \cap\left\{c^{i} b^{j}: j \geq p\right\}$, the set $S^{\prime}$ is an $\mathcal{L}^{*}$-class of $S$. For $S$ to be abundant 
it is necessary that $c^{p} b^{p} \in S$. There exist algorithms to construct the other finitely many $\mathcal{L}^{*}$-classes and $\mathcal{R}^{*}$-classes of $S$ from the finitely many elements of $S$ in columns $0, \ldots, p$ and the finite set $I$. The semigroup is abundant if and only if these finitely many $\mathcal{L}^{*}$-classes and $\mathcal{R}^{*}$-classes have idempotents.

The algorithms to check if a upper subsemigroup is abundant follows. We have seen it is necessary that $c^{p} b^{p} \in S$ for $S$ to be abundant. Then we can check if all $\mathcal{L}^{*}$-classes have idempotents with the algorithm in Figure 8, where $C$ is the set of indices of columns having elements in $F^{\prime} \cap S$.

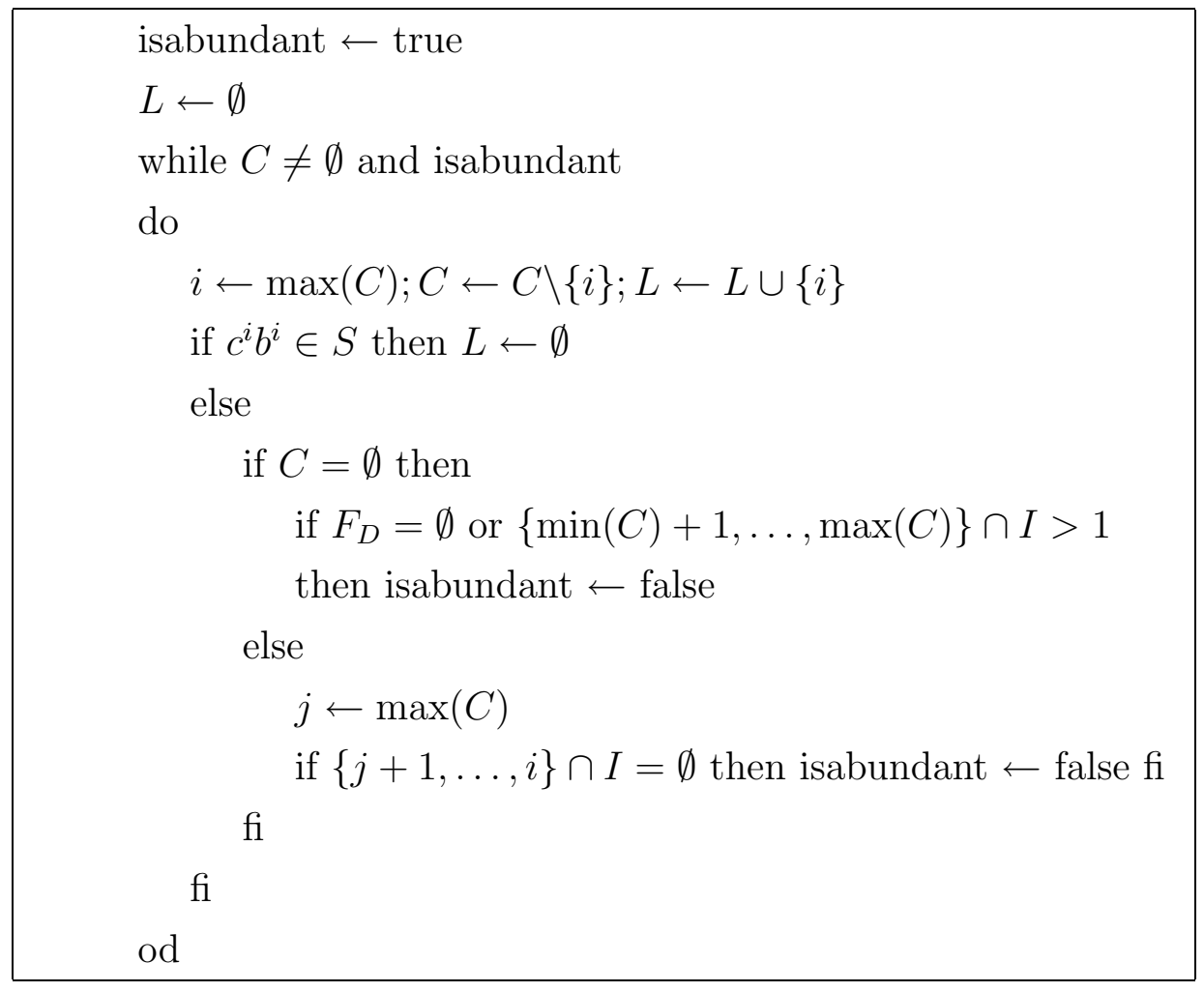

Figure 8: Algorithm to check if all $\mathcal{L}^{*}$-classes have indempotents

To check if all $\mathcal{R}^{*}$-classes have idempotents we can the use the algorithm in Figure 9.

Finitely generated lower subsemigroups are similar, just replacing rows by columns.

If $S$ is a non finitely generated upper subsemigroup, so with elements in an infinite number of rows, then there is no algorithm to check if $S$ is abun- 


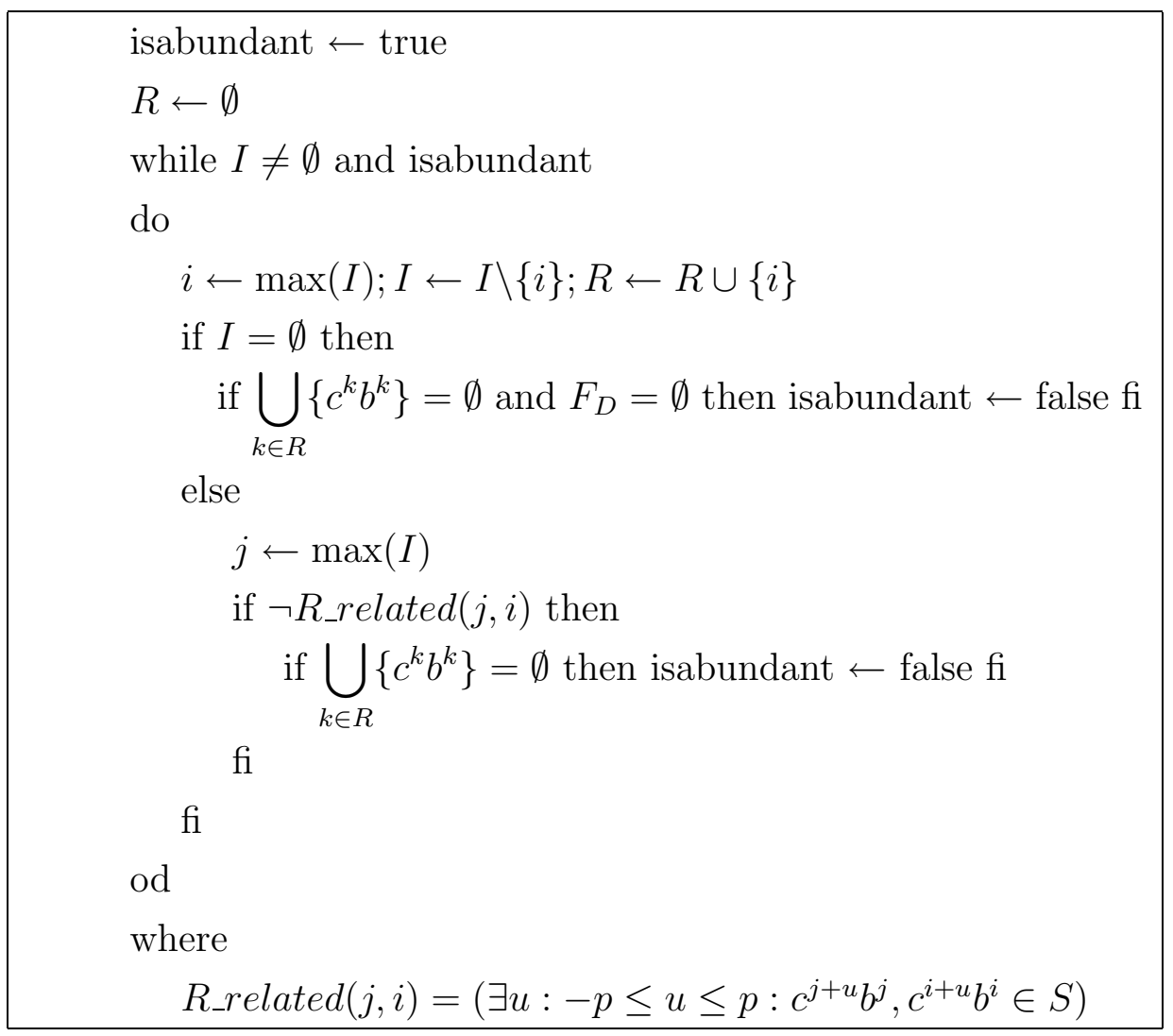

Figure 9: Algorithm to check if all $\mathcal{R}^{*}$-classes have indempotents 
dant. In fact, we cannot decide if $S$ is abundant, looking to finitely many rows, because we can always add a row without idempotent to an abundant semigroup obtaining a non abundant subsemigroup. For example, the semigroup $S=\left\{c^{i} b^{j}: 0 \leq i<p, j \geq i\right\}(p>0)$ is abundant and the semigroup $S \cup\left\{c^{p} b^{j}: j>p\right\}$ is not.

Of course there is a procedure to check if $S$ is not abundant. It suffices to construct the $\mathcal{L}^{*}$-classes $\left(\mathcal{R}^{*}\right.$-classes), which are unions of columns (rows), until a class without idempotent is found, using Lemma 3.4 (Lemma 4.6).

\section{TWO SIDED SUBSEMIGROUPS}

In general, a two-sided semigroup has the form $S=F_{D} \cup F \cup \Lambda_{I, p, d} \cup \Sigma_{p, d, P}$ (or the corresponding anti-isomorphic image) where $q, p \in \mathbb{N}_{0}$ with $q \leq p$, $d \in \mathbb{N}, I \subseteq\{q, \ldots, p-1\}$ with $q \in I, P \subseteq\{0, \ldots, d-1\}$ with $0 \in P$, $F_{D} \subseteq\left\{c^{i} b^{i}: i=0, \ldots, q-1\right\}, F \subseteq\left\{c^{i} b^{j}: q \leq i<p, i \leq j<p\right\}, \Sigma_{p, d, P}=$ $\left\{c^{p+r+u d} b^{p+r+v d}: r \in P ; u, v \geq 0\right\}$ (see [3]). Figure 10 shows an example of one of this subsemigroups.

We note that a two-sided semigroup of the form $F_{D} \cup \Sigma_{p, d, P}$ is regular (see [3]) and so abundant. Each of its $\mathcal{L}^{*}$-classes and $\mathcal{R}^{*}$-classes is contained in single row or column and all have an idempotent. We start by showing the following:

Proposition 6.1 Subsemigroups of the form $S=\Lambda_{I, p, d} \cup \Sigma_{p, d, P}$ (with $F_{D}=$ $F=\emptyset)$ are abundant.

Proof. If $I=\emptyset$ we have seen that $S$ is abundant, so we assume $I \neq \emptyset$. We begin by showing that two columns $i, j(i<j)$ such that, the set $\left\{c^{k} b^{k}\right.$ : $i \leq k \leq j\} \cap S$ is either empty or equal to $\left\{c^{i} b^{i}\right\}$, are $\mathcal{L}^{*}$-related. In fact, since $i, j \geq p$, the rows $i+1, \ldots, j$ are in $\Sigma_{p}$. A row $k$ in $\Sigma_{p}$ has elements from $S$ if and only if $c^{k} b^{k} \in S$. Hence $S$ has no elements in rows $i+1, \ldots, j$ and Lemma 3.5 can be applied.

Using this, and observing thet $0 \in P$ and so $c^{p} b^{p} \in S$, we see that every $\mathcal{L}^{*}$ class is a union of columns starting from a column with an idempotent together with all columns on its right hand side not having idempotents. Hence, every $\mathcal{L}^{*}$-class has an idempotent. 


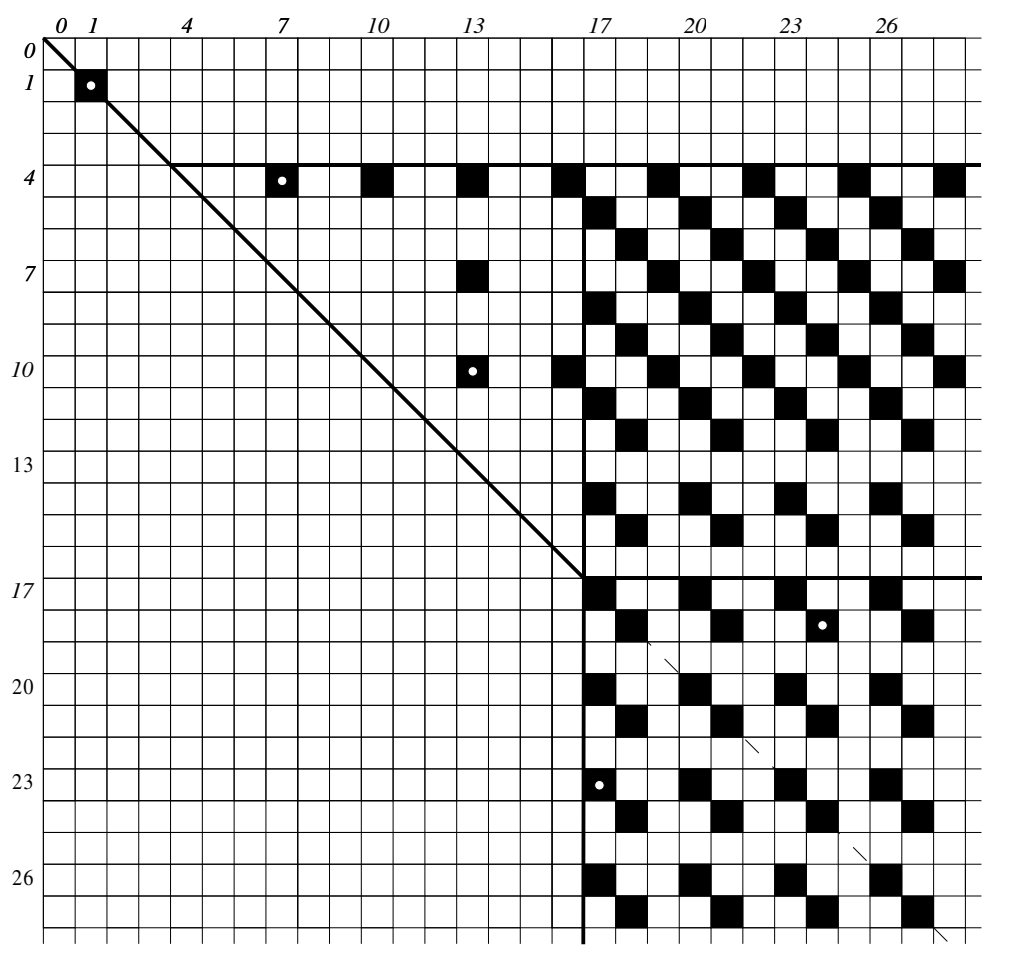

Figure 10: Two-sided subsemigroup generated by $\left\{c b, c^{4} b^{7}, c^{10} b^{13}, c^{18} b^{24}, c^{23} b^{17}\right\}$.

Each row in $p+1, p+2, \ldots$ with elements in $S$ is an $\mathcal{R}^{*}$-class with idempotent. The elements in $S \cap\left\{c^{i} b^{j}: 0 \leq i \leq p, j \geq p\right\}$ are $\mathcal{R}^{*}$-related because $S$ has no elements in columns $q, \ldots, p-1$ and Lemma 4.6 can be applied. Hence every $\mathcal{R}^{*}$-class has an idempotent.

Corollary 6.2 Every simple subsemigroup of the bicyclic monoid is abundant.

Proof. As shown in [3] these are the simple subsemigroups of the bicyclic monoid.

We consider now a general two sided semigroup $S=F_{D} \cup F \cup \Lambda_{I, p, d} \cup \Sigma_{p, d, P}$. For columns in $\Lambda_{I, p, d} \cup \Sigma_{p, d, P}$, the argument in the proof of Proposition 6.1 can be applied and so the classes with these elements have idempotents. Elements in columns $p-1$ and $p$ are note related because we can take $x=c^{p} b^{p+u d}, y=$ $c^{i} b^{i+v d}(i<p)$ in the conditions of Lemma 3.4. To form the $\mathcal{L}^{*}$-classes with 
the elements in columns $p-1, p-2, \ldots$ we just look to rows $i<p$ and so to $F_{D} \cup F \cup \Lambda_{I, p, d}$. Hence we just have to apply the algorithm in Figure 8, where $C$ is the set of indices of columns $i<p$ with elements in $S$.

Each row in $\Sigma_{p, d, P}$ is in a separate $\mathcal{R}^{*}$-class with idempotent. We note that rows $p$ and $p-1$ are not related if $F_{D} \cup F \neq \emptyset$. In fact, if $F_{D} \neq \emptyset$ then the elements $x=c^{p} b^{p}, y \in F_{D}$ are in the conditions of Lemma 4.6. And if $F \neq \emptyset$ we can also find two elements $x=c^{p-u d} b^{p}, y \in F$ in the conditions of Lemma 4.6. Hence, to check if all $\mathcal{R}^{*}$-classes have idempotents, we just have to look to rows in $F_{D} \cup F \cup \Sigma_{p, d, P}$ and columns $0, \ldots, p-1$ and we can use the algorithm in Figure 9. 


\section{ACKNOWLEDGEMENTS}

The first author acknowledges the support of the Fundação para a Ciência e a Tecnologia (Portugal) through Unidade de Investigação Matemática e Aplicações of University of Aveiro.

\section{REFERENCES}

[1] Adair, C. L. A generalization of the bicyclic semigroup. Semigroup Forum $1980,21,13-25$.

[2] Byleen, K.; Meakin, J.; Pastijn F. The fundamental four-spiral semigroup. J. Algebra 1978, 54, 6-26.

[3] Descalço, L.; Ruškuc, N. Subsemigroups of the bicyclic monoid. Internat. J. Algebra Comput. 2005, 15, 37-57.

[4] Descalço, L.; Ruškuc, N. Properties of the subsemigroups of the bicyclic monoid. Czechoslovak Mathematical Journal, to appear.

[5] Fountain, J. Abundant semigroups. Proc. Lond. Math. Soc. 1982, 44, 103129.

[6] Grillet, P. A. On the fundamental double four-spiral semigroup. Bull. Belg. Math. Soc. Simon Stevin 1996, 3, 201-208.

[7] Hogan, J. W. The $\alpha$-bicyclic semigroup as a topological semigroup. Semigroup Forum 1984, 28, 265-271.

[8] Howie, J. M. Fundamentals of Semigroup Theory; Oxford University Press: Oxford, 1991.

[9] Lawson, M. V. Inverse Semigroups, World Scientific: Singapore, 1998.

[10] Makanjuola, S. O.; Umar A. On a certain subsemigroup of the bicyclic semigroup. Comm. Algebra. 1997, 25, 509-519.

[11] Shevrin, L. N. The bicyclic semigroup is determined by its subsemigroup lattice. Bull. Belg. Math. Soc. Simon Stevin 1993, 67, 49-53.

[12] Shevrin, L. N.; Ovsyannikov A. J. Semigroups and Their Subsemigroup Lattices, Kluwer Academic Publishers, 1996. 